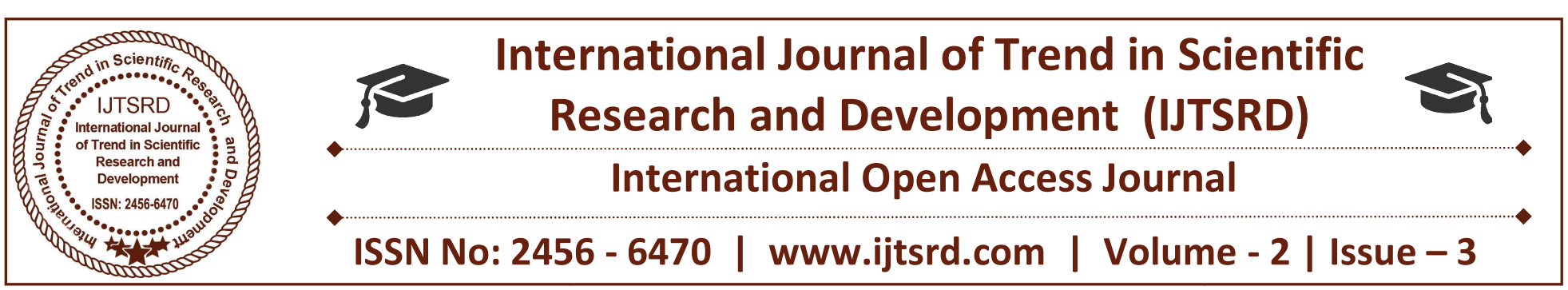

\title{
A Case Study on Projectile Motion
}

Vivek. S

Assistant Professor, Sri Krishna

Adithya College of Arts and

Science, Arivoli Nagar,

Coimbatore, Tamil Nadu, India

\author{
Rohini. R \\ B.Sc Maths, Sri Krishna Adithya \\ College of Arts and Science, \\ Arivoli Nagar, Coimbatore, \\ Tamil Nadu, India
}

\author{
Dhanya. G \\ B.Sc Maths, Sri Krishna Adithya \\ College of Arts and Science, \\ Arivoli Nagar, Coimbatore, \\ Tamil Nadu, India
}

\section{ABSTRACT}

In this paper, the question of the motion of a projectile thrown at an angle to the horizon is learned. With zero air drag force, the analytic resolution is well understood. The course of the projectile is a parabola. In situations of practical interest, such as jaculatory a ball with the event of the strike of the medium the square resistance law is generally used. In that casing the question likely does not have an true analytic resolution and therefore in most expert publications it is solution numerically. Analytic advance to the resolution of the question are not sufficiently professional. Meanwhile, resolve solutions are very handy, for a honest preparedness to win problems and are expressly costly for a qualitative analysis. That is why the representation of the missile summon with a uncombed rough resolve formula under the quadratic air resistance instant big methodological interest. Lately these formulas have been procure. These formulas bestow us to obtain a finished divisive narration of the problem. This delineation perfect divisive formulas for shape the basic eight parameters of missile movement. Analytical formulas have been infer for the six fundamental official dependences of the statement including the trajectory equality in Cartesian coordinates. Also this description contains the judgment of the optimal throwing angle and maximum frequent of the motion. In the destitution of publicity resistance, all these relations transfer into well-understood formulas of the hypothesis, speculation of the parabolic motion of the projectile. The proposed analytical discharge oppose from other solutions by clearness of formulas, comfortableness of use and high fidelity (relation hallucination is near 1-2 $\%$ ). The motion of a baseball is instant as an example.
The converse formulas make it possible to carry out an resolvent examination of the motion of a projectile in a medium with resistance in the highway it is done in the casing of no drag.

Keywords: spherical object, quadratic drag force, analytical formula, relative error

\section{Introduction}

The problem of the motion of a detail mass (projectile) thrown at an angle to the circle in midair has a $g$ history. It is one of the great humanistic problems that lead the dynamics as knowledge. The amount of works devoted to this employment is vast. It delineatea elemental of many prefatory courses of physics. With no trail, the analytic resolution of this question is well known. The course of the stage assemble is a parabola. In this notes, an analytic appropinquate is necessity for the scrutation of the projectile motion in a medium with tetragonal resistance. The opposed of the immediate product is to give simple formulas for the sense of the projectile trajectories under the course with quadratic intelligence resistance. These formulas are advantageous even for first-year undergraduates. The question of the motion of a projectile in midair has stirred up interest of authors (Cohen et al., 2014; Kantrowitz \& Neumann, 2013; Borghi, 2013). For the construction of the separative solutions various methods are application - both the traditive approximate (Benacka, 2010; Vial, 2007; Parker, 1977; Erlichson, 1983; Tan, Frick \& Castillo, 1987)], and the modern methods (Yabushita, Yamashita \& Tsuboi, 2007). All proposed approximate analytical solutions are rather complicated and uncomfortable 
for educational aim. This is why the description of the projectile course by means of a single approximate analytical formula under the square mien resistance is of powerful methodological and instructive significance. In (Chudinov, 2002; 2004; 2013) comparatively harmless approximate analytical formulas have been possess to ponder the motion of the projectile in a medium with a quadratic comfit violence. In this article, these formulas are interest to solve the humanistic problem of maximizing the projectile distance. From now on, the word "appoint mass" contemptible the center of mass of a smooth rounded object of limited circle $\mathrm{r}$ and trial-sectional region $\mathrm{S}=\pi \mathrm{r} 2$. The provision of applicability of the quadratic resistance law are esteem to be fulfilled, i.e. Reynolds scalar Re sleep within $1 \times 103<\operatorname{Re}<2 \times 105$. These values correspond to the missile motion velocity, fabling in the range between $0.25 \mathrm{~m} / \mathrm{s}$ and 53 $\mathrm{m} / \mathrm{s}$.

\section{Equations of Point Mass Motion and Analytical} Formulas for Basic Parameters

Suppose that the force of gravity concern the appoint mass together with the force of air resistance $\mathrm{R}$ (see Fig. 1). Air resistance force is corresponding to the square of the celerity of the point body and is addressed opposite the fleetness vector. For the convenience of further calculations, the starch cascade will be written as $2 \mathrm{R} \operatorname{mgkV}=$. Here $\mathrm{m}$ is the mass of the projectile, $\mathrm{g}$ is the acceleration due to gravity, $\mathrm{k}$ is the proportionality agent. Vector equation of the guide of the point mass has the beauty

$m w=m g+R$,

where $\mathrm{w}$ - acceleration vector of the point mass. Differential equations of the motion, commonly used in ballistics, are as follows (Okunev, 1943)

$$
\begin{aligned}
& \frac{d V}{d t}=-g \sin \theta-g k V^{2}, \frac{d \theta}{d t}=-\frac{g \cos \theta}{V}, \frac{d x}{d t}=V \cos \theta \\
& \frac{d y}{d t}=V \sin \theta
\end{aligned}
$$

Here $\mathrm{V}$ is the velocity of the appoint sum, $\theta$ is the angle between the tangent to the course of the moment bulk and the flat, $\mathrm{x}, \mathrm{y}$ are the Cartesian coordinates of the instant bulk, $\mathrm{k}$ is

$$
k=\frac{p_{a c_{d} S}}{2 m g}=\frac{1}{V^{2}}=\text { conts },
$$

$P_{a}$ is the air density, cd is the drag factor for a sphere, $\mathrm{S}$ is the misfortune-section scope of the goal, and $\mathrm{V}_{\text {_t }}$ term is the termination celerity. The first two equations of the system (1) personate the projections of the vector equation of guide on the tangent and main analogical to the course; the other two are kinematic relations connecting the projections of the fleetness vector point bulk on the shaft $\mathrm{x}$, $\mathrm{y}$ with derivatives of the coordinates.

\section{Figure 1: Basic Motion Parameters}

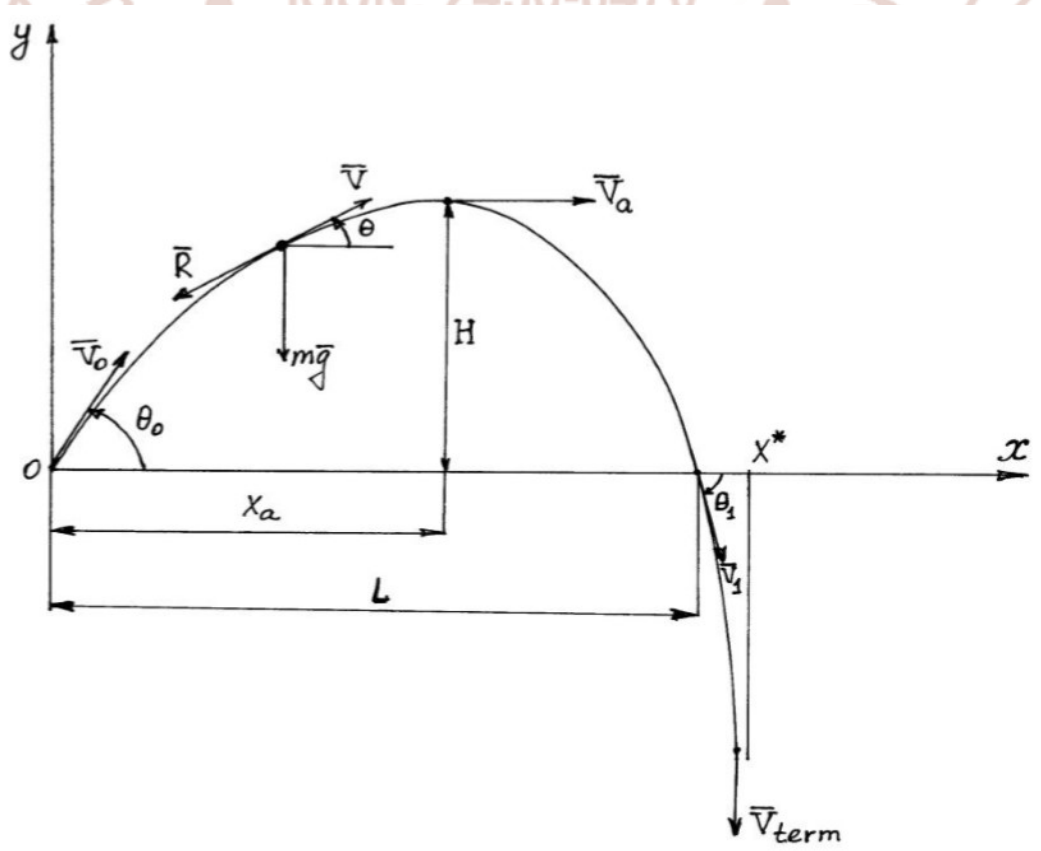


The well-known solution of system (1) consists of an explicit analytical dependence of the velocity on the slope angle of the trajectory and three quadratures $V(\theta)=\frac{V 0 \cos \theta}{\cos \theta \sqrt{1+k V_{0}^{2} \cos ^{2} \theta_{0}\left(f\left(\theta_{0}\right)-f(\theta)\right.}}$

$\int(\theta)=\frac{\sin \theta}{\cos 2 \theta}+$ in $\operatorname{tg}\left(\frac{\theta}{2}+\frac{\pi}{4}\right)$

$T=t_{0-} \frac{1}{g} \int_{\theta_{0}}^{\theta} \frac{V}{\cos ^{\theta}} d \theta, x=x_{0}-\frac{1}{g} \int_{\theta_{0}} V^{2} d \theta$ $\mathrm{y}=y_{0}-\frac{1}{g} \int_{\theta_{0}}^{0} V^{2} \operatorname{tg} \theta d \theta$

Here V0 and $\theta$ are the commencing importance of the fleetness and the slope of the trajectory respectively, $\mathrm{t} 0$ is the incipient importance of the period, $\mathrm{x} 0, \mathrm{y} 0$ are the drop cap values of the coordinates of the instant bulk (most often approve t $0_{-}=\mathrm{x}_{-} \mathrm{O}=\mathrm{y}_{-} \mathrm{O}=0$ ). The derivative of the formulas (2) is shown in the wellknown monograph (Timoshenko \& Young, 1948). The integrals on the right-hand sides of formulas (3) cannot be expressed in limit of simple performance. Hence, to shape the variables $t, x$ and $y$ we must either complete system(1) numerically or appraise the precise integrals (3). Comparatively single approximate analytical formulas for the principal parameters of tendency of the projectile are deduce in (Chudinov, 2002, 2004). The four parameters address to the top of the trajectory, four - to the stage of drip. We will give a consummate concise of the formulas for the maximum height of ascent of the point mass $\mathrm{H}$, motion time $\mathrm{T}$, the fleetness at the trajectory tip $\mathrm{Va}$ $=\mathrm{V}(0)$, stampede range $\mathrm{L}$, the repetition of ascent ta, the absciss of the trajectory pinnacle xa, impact angle with esteem to the horizontal $\theta 1$ and the final speed V1 (see Fig. 1). These formulas are sum in the right column of Table 1. In the port column of this Table 1 homogenous formulas of the allegorical theory are immediate for illustration.

Analytical Formulas for the Main Parameters

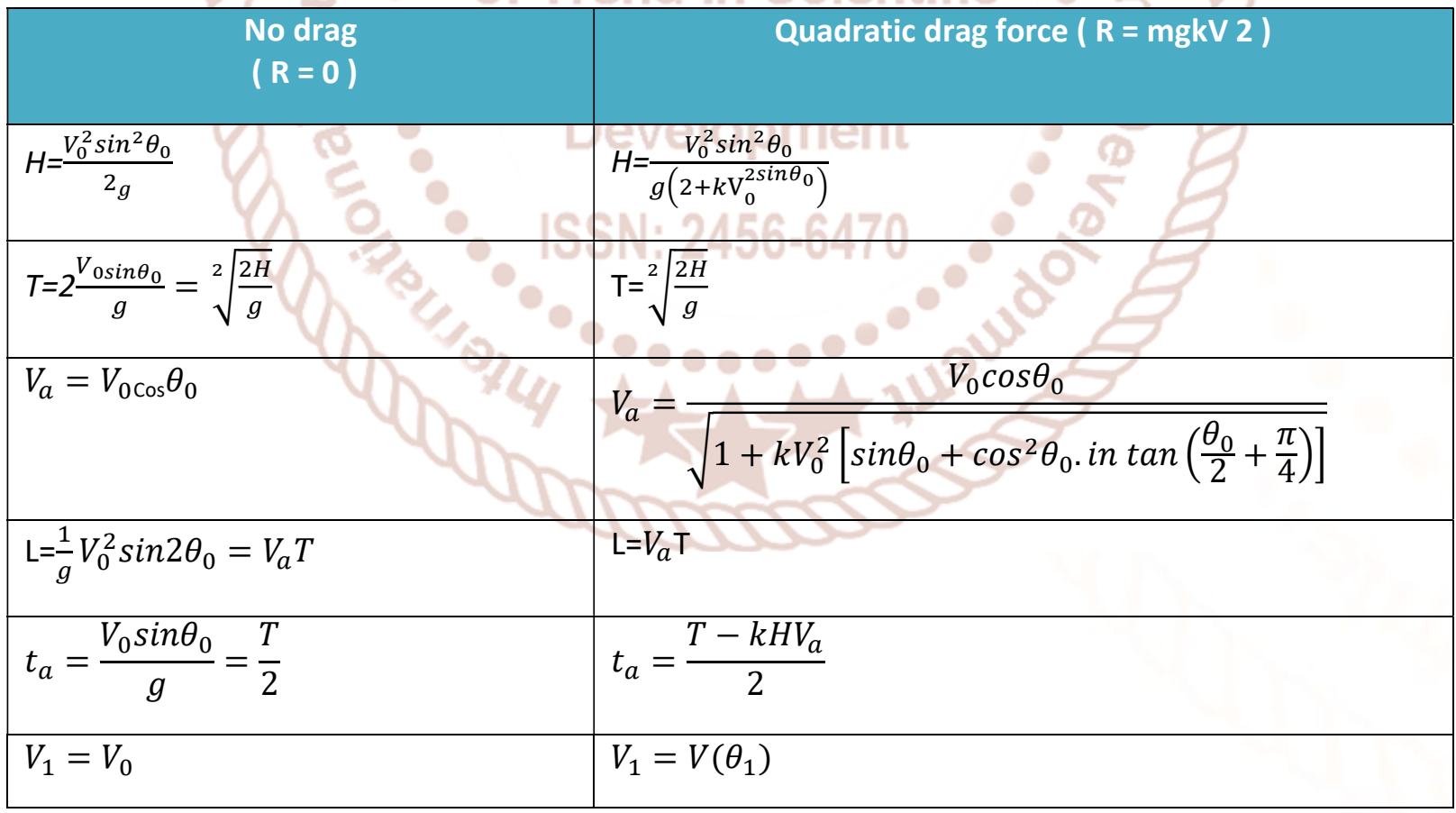

With zero drag $(\mathrm{k}=0)$, these formulas go over into the wary formulas of the point mass parabolic motion theory. All movement characteristics relate by these formulas are functions of opening requisite of throwing V0, 00 . Proposed formulas have a bounded region of application. We present the symbolology 2
$0 \mathrm{p} \mathrm{kV \_} 0^{\wedge} 2$. The dimensionless parameter $\mathrm{p}$ has the profession physical object - it is the ratio of air resistance to the moment of the projectile at the opening of the motion. The principal characteristics of the motion $\mathrm{H}, \mathrm{T}, \mathrm{Va}, \mathrm{L}$, xa have precision to within 2 
- $3 \%$ for values of the pierce angle, for commencing speed and for the parameter $\mathrm{p}$ from ranges

$0^{\circ} \leq \theta 0 \leq 70^{\circ}, \quad 0050 \mathrm{~V} \square \square \mathrm{m} / \mathrm{s}, \quad 0 \leq \mathrm{p} \leq 1.5$.

For a baseball the typical values of the drag force coefficient $\mathrm{k}$ are near $0.0005 \div 0.0006 \mathrm{~s} 2 / \mathrm{m} 2$, maximal commencing speed is circularly $50 \mathrm{~m} / \mathrm{s}$ (Cohen et al., 2014; Tan et al., 1987). Therefore the converse formulas are competent for the qualitative and quantitative narrative of the motion of the baseball and other resemblant goal. These formulas, in devote, occasion it likely to obtain a uncompounded divisive formula for the principal functional relationship of the proposition $\mathrm{y}(\mathrm{x})$ (Chudinov, 2002). In the want of air resistance, the trajectory of a point mass is a parabola. The equation of the trajectory can be scriptory in two constitution. It can be scriptory in bound of the opening station of jaculatory $V_{0}, \theta_{0}$ (first figure). It can also be written in terms of the motion parameters $\mathrm{H}, \mathrm{L}$, xa (second form)

$\mathrm{Y}(\mathrm{x})=\mathrm{x} \cdot \tan \theta_{0}-\frac{g X^{2}}{2 V_{0}^{2 \operatorname{Cos}^{2} \theta_{0}}}=\frac{H x(L-X)}{x_{a}^{2}}$

The course is symmetric with respect to the greatest. When the item mass is under a drag force, the course becomes asymmetric. The top of the trajectory is divide towards the point of incident. In title, a vertical asymptote appearance near the trajectory. Taking these circumstances into narrative, the performance $\mathrm{y}(\mathrm{x})$ may be arrange using parameters $\mathrm{H}, \mathrm{L}$, $\mathrm{xa}$ as (Chudinov, 2002)

$\mathrm{Y}(\mathrm{x})=\frac{H_{X}(L-X)}{X_{a}^{2+}\left(L-2 X_{a}\right) x}$

The constructed dependence $\mathrm{y}(\mathrm{x})$ provides the shift of the apex of the trajectory to the right and has a vertical asymptote. In the case of no drag $\mathrm{L}=2 \mathrm{xa}$ and formula (5) goes over to formula (4). We note the remarkable property of formula (5). We insert the exact values of the parameters $\mathrm{L}, \mathrm{H}, x_{a}$, obtained by numerical integration of system (1), into formula (5). Then the numerical trajectory and the analytical trajectory constructed by means of formula (5) are identical. This means that formula (5) approximates absolutely precisely the projectile's trajectory which is numerically constructed using system (1) at any values of the initial conditions $V_{0}, \theta_{0}$. The some transformation of the proposed formulas (Chudinov, 2013) makes it possible to improve the accuracy of calculating the main parameters. Now it is possible to construct the trajectory in the entire range of launch angles and at values of the initial velocity and the parameter $\mathrm{p}$

$0^{\circ} \leq \theta_{0} \leq 90^{\circ}, 0 \leq V_{0} \leq 80 \mathrm{~m} / \mathrm{s}, \quad 0 \leq \mathrm{p} \leq 4$.

As an represent of the use of the speak formulas from the Table 1 and of formula (5) we calculated the movement of a baseball with the following initial station

$V_{0}=40 \mathrm{~m} / \mathrm{s} \quad, \theta_{0}=45^{\circ}, k=0.000625 \mathrm{~s}^{2} / \mathrm{m}^{2}, g=$ $9.81 \mathrm{~m} / \mathrm{s}^{2}$

Comparison of Numerical and Analytical Calculations

\begin{tabular}{|l|l|l|l|}
\hline Parameter & $\begin{array}{l}\text { Analytical } \\
\text { value }\end{array}$ & $\begin{array}{l}\text { Numerical } \\
\text { value }\end{array}$ & $\begin{array}{l}\text { Error } \\
(\%)\end{array}$ \\
\hline $\mathrm{H}(\mathrm{m})$ & 30.1 & 29.8 & 1.1 \\
\hline $\mathrm{T}(\mathrm{s})$ & 4.96 & 4.91 & 1.0 \\
\hline $\mathrm{Va}(\mathrm{m} / \mathrm{s})$ & 19.3 & 19.3 & 0 \\
\hline $\mathrm{L}(\mathrm{m})$ & 95.7 & 96.1 & -0.4 \\
\hline ta (s) & 2.30 & 2.31 & -0.4 \\
\hline xa (m) & 53.7 & 53.0 & 1.3 \\
\hline$\theta 1$ (degree) & $-58.6^{\circ}$ & $-57.3^{\circ}$ & 2.2 \\
\hline
\end{tabular}

The terminate of calculations are recorded in Table 2 and are shown in Figure 2. The help atlantes restrain the importance calculated by deliver formulas from the Table 1 . The third atlantes show the importance of parameters obtained by numeral integration of the motion equations (1) by the arrangement RungeKutta process. The deviations from the correct values of parameters are shown in the fourth column of the Table 2. Tabulated data show that the importance of basic parameters of the projectile motion (flight range $\mathrm{L}$, tendency time $\mathrm{T}$, prominence $\mathrm{H}$ ) adapted by divisive formulas contend from the exact values no more than $1 \%$. An round trajectory (5) is compile with the help of parameters H, L, xa. It is shown in Figure 2 (dotted line). The indistinct important rope in Figure 2 is possess by numerical integration of system (1) with the befriend of the 4-th order Runge-Kutta method. As can be accomplished from Figure 2, the divisive solution (formula (5)) and a numeral resolution are almost the same. The frustrate line in Figure 2 is erect in the destitution of mien resistance 


\section{The Graph of the Trajectory $y=y(x)$}

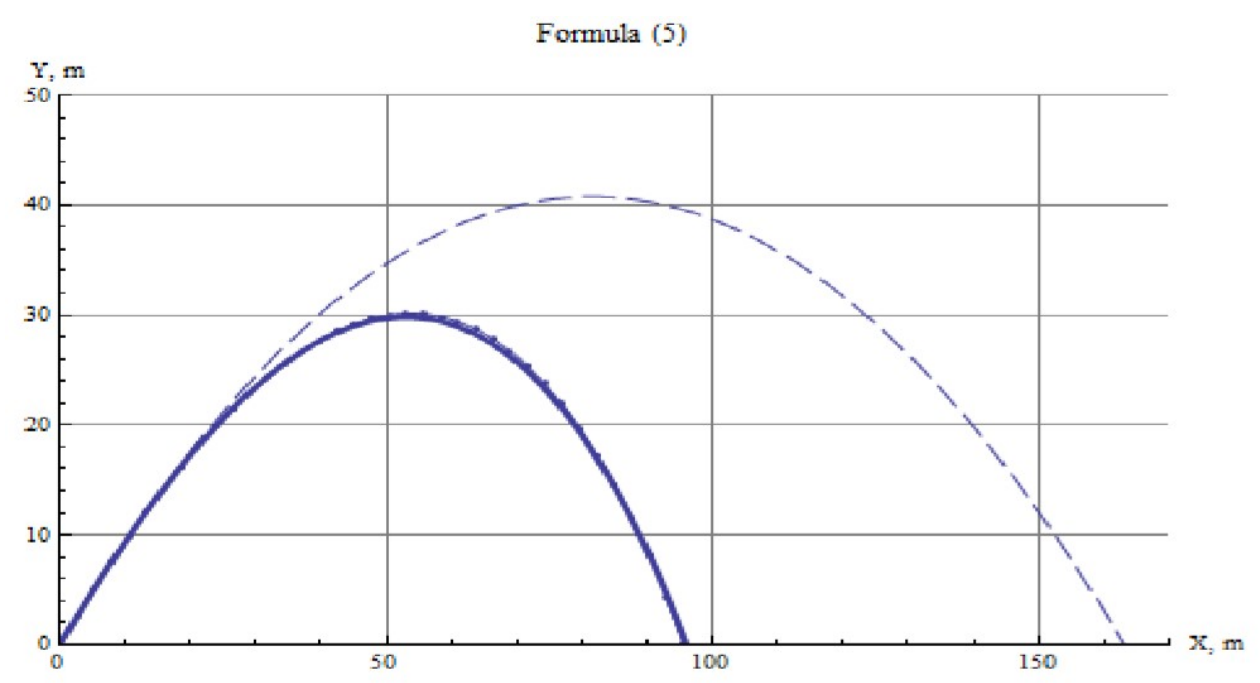

One of the most necessary aspects of the projectile movement problem is determination of an optimal viewpoint of jaculatory of a point number which provides the maximum range. Let us make usefulness of formulas from the Table 1 and (5) to resolve the problem of optimization of motion parameters
Determination of an Optimum Throwing Angle and Maximum Range

Let the point of incidence $\mathrm{A}$ be on a horizontal straight line defined by the equation const $y=$ $\mathrm{y}_{1}=\mathrm{const}$

\section{Statement of the Optimization Problem}

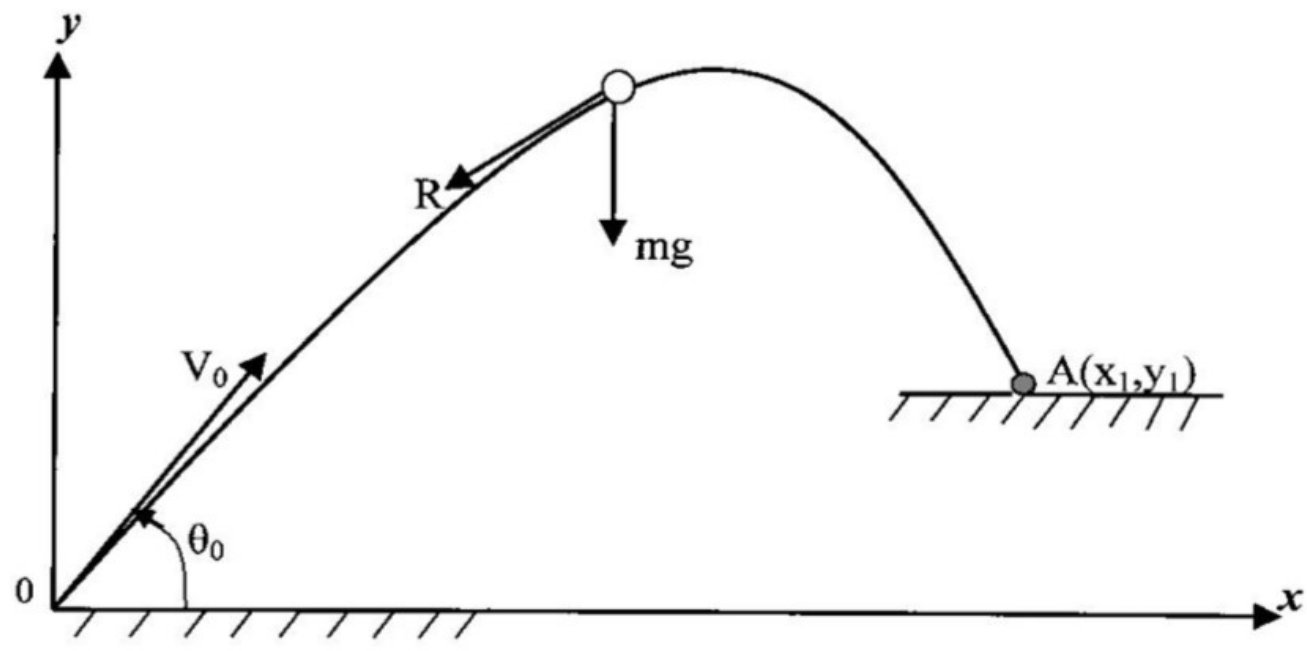

To solve the problem, let us companion use of the appoint mass trajectory equality (5). In this equality, the tendency characteristics $\mathrm{H}, \mathrm{L}, \mathrm{x}_{\mathrm{a}}$ are duty of initial station of throwing, $V_{0} \theta_{0}$. They are explain by formulas from the Table 1 . Let us change $y_{1}$ in the sinistral-hand part of equality (5) and resolve it for variablex. Thus, we must get the fleeing frequent formula for the casing under contemplation

\section{SUMMARY}

The proposed approach based on the usefulness of analytic formulas mate it possibility to disintricate way a qualitative analysis of the motion of a projectile with the air draw taken into reckoning. All basic parameters of guide and various problems of optimization are relate by unadorned analytical formulas confine rudimental functions. Moreover, numeral values of the sought variables are resolute with an comfortable propriety. It can be accomplish even on a authoritative calculator. Lately some creator 
(Warburton \& Wang, 2004; Stewart, 2005; Hu et al., 2012) have used the Lambert W service to study the projectile motion with resistance. But this relatively "new" function is not profitable on a calculator. Special algorithms are required to compute this service. Thus, discourse formulas make it possibility to study projectile motion with tetragonal drag force even for first-year undergraduates. In end, we hope that efforts to prevail an analytical release to this question will be extended and will achieve new exact and effective solutions.

\section{CONCLUSION}

A projectile that does not contain an cordite charge or any other good of instruct is condition a kinetic projectile, motory action weapon, seepy Life warhead, dynamic warhead or motory penetrator. Typical kinetic energy weapons are blunt projectiles such as cliff and round shots, pointed ones such as directed edge, and little pointed ones such as ball. Among projectiles that do not hold explosives are those launched from railguns, coilguns, and quantity drivers, as well as active force penetrators. All of these weapons work by obtain a lofty muzzle velocity, or commencing velocity, collectively up to (hypervelocity), and collide with their aim, translate their kinetic energy into mischievous shock waves and heat. Other types of animated arm are accelerated over era by a rocket engine, or by gravity. In either plight, it is the active strength of the projectile that consume its target.

\section{REFERENCES}

1. Baće, M., Ilijić, S., Narancić, Z., Bistricić, L. (2002).The envelope of projectile trajectories. European Journal of Physics. 23, 637-642.

2. Benacka, J. (2010). Solution to projectile motion with quadratic drag and graphing the trajectory in spreadsheets. International Journal of Mathematical Education in Science and Technology. 41, 373-378.

3. Borghi, R. (2013). Trajectory of a body in a resistance medium: an elementary derivation. European Journal of Physics. 34, 359-370.

4. Chudinov, P.S. (2002).The motion of a heavy particle in a medium with quadratic drag force.International Journal of Nonlinear Sciences and Numerical Simulation. 3, 121-129.

5. Chudinov, P.S. (2004). An optimal angle of launching a point mass in a medium with quadratic drag force. In Proceedings of 21-th International Ballistics Symposium (Adelaide, South Australia, April 19 - 23, 2004, Vol. 1, 511517).

6. Chudinov, P.S. (2004).Analytical investigation of point mass motion in midair.European Journal of Physics. 25, 73-79. 